\title{
Comparative efficacy and safety of phosphodiesterase-5 inhibitors with selective serotonin reuptake inhibitors in men with premature ejaculation
}

\section{A systematic review and Bayesian network meta-analysis}

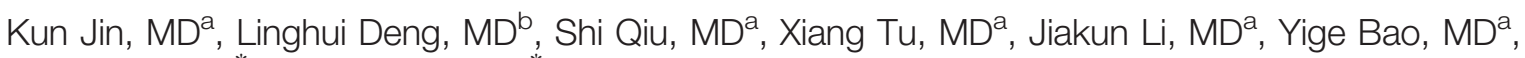
Lu Yang, MD ${ }^{a, *}$, Qiang Wei, MD ${ }^{a, *}$

\begin{abstract}
Background: We performed the network meta-analysis (NMA) and systematic review involved all evidence from relevant trials to compare the efficiency and safety of various types of selective serotonin reuptake inhibitors (SSRI) and phosphodiesterase-5 inhibitors (PDE5i) in patients with premature ejaculation (PE).

Methods: We conducted comprehensive searches of peer-reviewed and grey literature. PubMed, the Cochrane Library Central Register of Controlled Trials, Embase were searched for randomized controlled trials published up to June 1, 2017. The primary outcome was intravaginal ejaculation latency time (IVELT) and adverse effects (AEs). We performed pairwise meta-analyses by random effects model and network meta-analysis by Bayesian model. We used the GRADE framework to assess the quality of evidence contributing to each network estimate.

Results: Of 3046 titles and abstracts initially identified, 17 trials reporting 5739 participants were included. Considering IVELT in the NMA, paroxetine plus sildenafil and sildenafil alone are both superior to placebo (MD: 1.75, 95\% Crl: 0.05 to 3.78 ; MD 1.43, 95\% Crl 0.003 to 2.81). Sildenafil is superior to sertraline (MD: $1.63,95 \% \mathrm{Crl}: 0.10$ to 2.79). Considering AEs, placebo demonstrated obviously lower risk comparing to paroxetine, sildenafil and paroxetine plus sildenafil (OR 0.20, 95\% Cl: 0.05 to 0.52 ; OR 0.23, 95\% Cl: 0.04 to 0.80; OR 0.45, 95\% Cl: 0.01 to 0.92). Compared with tadalafil plus paroxetine, dapoxetine showed significantly less AEs (OR 0.23, 95\% Cl 0.02 to 0.96).

Conclusions: Our study concluded that although paroxetine plus sildenafil and sildenafil alone both demonstrated significant IVELT benefit compared with placebo, significant increase of AEs risk was also observed. Furthermore, sildenafil alone was superior to sertraline in efficacy with comparable tolerability.
\end{abstract}

\section{Editor: Guanyi Zhang.}

Kun Jin, Linghui Deng, and Shi Qiu: These authors have contributed equally to this work. We synthesize the best available evidence to inform premature ejaculation treatment strategies.

In the absence of head-to-head trials, this is the first systematic review and network meta-analysis to provide a comprehensive estimate of relative efficacy and safety of premature ejaculation treatment strategies.

We include only English-language peer-reviewed random randomized controlled trials making it possible that some relevant articles were not included.

The considerable amount of heterogeneity between included studies was not fully explained by the variables examined.

This study was supported by the Prostate Cancer Foundation Young Investigator Award 2013, the National Natural Science Foundation of China (Grant No.81300627, 81200551, 81270841, 81460148, 81500522 and 81370855), Programs from Science and Technology Department of Sichuan Province (Grant No. 2013SZ0006 and 2014JY0219). International Cooperation Fund of Sichuan Science and Technology Program (2017HH0063). China Postdoctoral Science Foundation (2017M612971).

The authors have no conflicts of interest to disclose. Provenance and peer review Not commissioned; externally peer reviewed.

Data sharing statement Additional data are presented in supplemental files.

Supplemental Digital Content is available for this article.

${ }^{a}$ Department of Urology, Institute of Urology, West China Hospital of Sichuan University, ${ }^{b}$ Stroke Clinical Research Unit, Department of Neurology, West China Hospital of Sichuan University, Chengdu, Sichuan, PR China.

* Correspondence: Qiang Wei, Department of Urology, Institute of Urology, West China Hospital of Sichuan University, No. 37, Guoxue Alley, Chengdu, Sichuan, PR China (e-mail: weiqiang163163@163.com); Lu Yang, Department of Urology, Institute of Urology, West China Hospital of Sichuan University, No. 37, Guoxue Alley, Chengdu, Sichuan 610041, P.R. China (e-mail: wycleflue@163.com).

Copyright (C) 2018 the Author(s). Published by Wolters Kluwer Health, Inc.

This is an open access article distributed under the terms of the Creative Commons Attribution-Non Commercial License 4.0 (CCBY-NC), where it is permissible to download, share, remix, transform, and buildup the work provided it is properly cited. The work cannot be used commercially without permission from the journal.

Medicine (2018) 97:49(e13342)

Received: 14 June 2018 / Accepted: 29 October 2018

http://dx.doi.org/10.1097/MD.0000000000013342 
Abbreviations: $\mathrm{AEs}=$ adverse effects, $\mathrm{Cl}=$ confidence intervals, $\mathrm{Crl}=$ credibility intervals, IVELT = intravaginal ejaculation latency time, $\mathrm{MD}=$ mean difference, $\mathrm{NMA}=$ network meta-analysis, $\mathrm{OR}=$ odds ratio, PDE5i $=$ phosphodiesterase-5 inhibitors, $\mathrm{PE}=$ premature ejaculation, $\mathrm{RCT}=$ randomized controlled trials, SSRI = selective serotonin reuptake inhibitors.

Keywords: phosphodiesterase type 5 inhibitor, premature ejaculation, selective serotonin reuptake inhibitor

\section{Introduction}

Premature ejaculation (PE) is a common adult male sexual disorder, with the prevalence rates of $20 \%$ to $30 \% .{ }^{[1]}$ According to the newly International Society for Sexual Medicine (ISSM) Guidelines, PE is defined as "Ejaculation that always occurs less than $1 \mathrm{~min}$ of vaginal penetration from the first sexual experience (lifelong PE), or a clinically significant and bothersome reduction in latency time, often $\leq 3 \mathrm{~min}$ (acquired PE)" ${ }^{[2]}$ It is associated with 'marked distress or interpersonal difficulty ${ }^{[3]}$ affecting sexual enjoyment and confidence, relationships of both partners and other aspects of an adult man's life. ${ }^{[4,5]}$ Intravaginal ejaculation latency time (IVELT) is regarded as the most common and useful treatment standard, for the reason of its sensitivity in measuring the efficacy of PE treatment. ${ }^{[6,7]}$

At present, medication therapy of PE includes selective serotonin reuptake inhibitor (SSRI) therapy (citalopram, sertraline, fluoxetine, dapoxetine or paroxetine), phosphodiesterase type 5 inhibitor (PDE5i) therapy (tadalafil or sildenafil), topical desensitizing agents (prilocaine or lidocaine) and other agents (tramadol or pindolol). Reports showed that oral intake of SSRI appeared as effective for patients with $\mathrm{PE}{ }^{[8]}$ resulting from its function of increasing the time IVELT. Except for efficacy, SSRI is recommended by AUA and International Society of Sexual Medicine guidelines because of its character of well-tolerated (eg, less anorexia, anejaculation, gastrointestinal upset and reduced libido). ${ }^{[9]}$

The choice of first line treatment is best addressed by direct comparisons of treatment regimens in high quality studies, but such studies considering SSRI combined with PDE5i do not yet exist for PE. Previous systematic reviews and meta-analyses have relied on direct comparisons: dapoxetine in different dose versus placebo, ${ }^{[10,11]}$ SSRI plus PDE5i versus placebo, ${ }^{[12]}$ SSRI, PDE5i versus the combination of SSRI plus PDE5i, ${ }^{[13]}$ SSRI versus PDE5i, ${ }^{[14]}$ tramadol versus placebo, ${ }^{[15]}$ SSRI, PDE5i, tramadol versus placebo. ${ }^{[16]}$ These reviews restricted to the overall variety (eg, SSRI, PDE5i or combination of SSRI plus PDE5i). Two metaanalysis had limitations as they focused on only a select number of interventions (dapoxetine or tramadol), which limited the evidence to a fraction of that available. Furthermore, in the absence of head-to-head trials, their relative efficacy and safety were unknown. We performed a pairwise meta-analysis and Bayesian network meta-analyses (NMA), comparing the relative efficacy and safety of PE treatment strategies.

\section{Methods}

\subsection{Search strategy}

Medline, Embase, the Cochrane central register of controlled trials, and the reference lists of the retrieved studies were searched to identify RCTs studies. A search strategy using the medical subject heading and text keywords: "premature ejaculation" was used (see online supplementary appendix 1, http://links.lww. com/MD/C673). The latest search was completed on 31 August, 2017. The previous published systematic reviews and reference lists of retrieved publications were also reviewed for additional studies. Ethical approval was not necessary for our study because all the participants were from others' RCT and our NMA did not involve information collection of patients.

\subsection{Study selection}

Following the principles of PRISMA (Preferred Reporting Items for Systematic Reviews and Meta-Analyses), we established the inclusion criteria before searching. ${ }^{[17]}$ Two reviewers (KJ and SQ) included all prospective randomized controlled trials (RCT) comparing one or more common pharmaceutical treatment with SSRIs combined with PDE5I, SSRIs monotherapy, PDE5I monotherapy or placebo for the PE patients. Studies that met the following criteria were finally included:

(1) trials were conducted in a homogenous group of PE patients;

(2) the interventions compared included at least 2 of the 4 treatment options;

(3) the outcomes included IVELT, satisfactory score and/or adverse effects (AEs);

(4) full-text original articles. Studies were excluded if they were non-English, review, non-randomized intervention, and study design.

We also excluded crossover trials and quasi-randomized studies. If duplicate studies were found, only the publication with the most complete data was used. Using the reference manager software Endnote, we identified and remove the duplicate records. Next step, 2 members (KJ and SQ) of our team independently scanned each title and abstract. If necessary, 2 study team members performed independent full-text reviews. After scanning all potentially relevant articles, 2 members of our study team discussed to consensus. Any discrepancies in the study inclusion were resolved by consulting the senior authors (QW).

\subsection{Data extraction}

Data were extracted by 2 reviewers (S.Q and K.J) from original trial using a specifically designed form that collected information on first author, publication year, the number of patients, definition of PE, patient characteristics (age, acquired/lifelong PE), details of treatment options and outcomes. We chose IVELT as the primary outcome, defined as the time that intercourse lasts from initiation of vaginal penetration to ejaculation. It was mainly measured by a stopwatch and expressed in minutes. Secondary outcomes were satisfactory score and AEs, each patient recorded their satisfaction with sexual intercourse before and post the treatment. We mainly concentrated on the change in posttreatment of IVELT and satisfactory score over the baseline. The AEs reported in each treatment intervention mainly including headache and dizziness, fatigue, decreased libido, gastrointestinal upset, palpitation, nasal congestion, erectile dysfunction and flushing. Disagreements were resolved by a third reviewer (Q.W).

\subsection{Assessment of risk of bias}

Two reviewers (K.J and S.Q) assessed studies quality using the methodology and categories on the basis of the Cochrane 
Collaboration Handbook. ${ }^{[18]}$ Concisely, the tool for assessing risk of bias includes 7 specific domains: random sequence generation, allocation concealment, blinding of participants and investigators, blinding of outcome assessment, incomplete outcome data, selective reporting, and other sources of bias. Each domain is assigned a judgement relating to the risk of bias for that study classified as low risk, high risk, or unclear. We presented risk of bias graphs by Review Manager 5.3 software.

\subsection{Data synthesis and statistical analysis}

We performed pair-wise meta-analysis in random effects model initially. We expressed the results as mean difference (MD) with 95\% confidence intervals (CI) for continuous outcomes (variation of IVELT or Satisfactory Score), and the odds ratio (OR) with $95 \%$ confidence intervals (CI) for discontinuous outcomes (AEs). The Cochran $Q$ test and the $\mathrm{I}^{2}$ statistic were used to evaluate the statistical heterogeneity among studies. A $P$ value of .05 or less for the $Q$ test or an $\mathrm{I}^{2}$ greater than $50 \%$ was suggestive of substantial study heterogeneity. Random-effects Bayesian NMA was performed for indirect and mixed comparisons using Markov chain Monte Carlo methods in WinBUGS version 1.4.3. ${ }^{[19]}$ We report the resultant effect as MD/OR with corresponding $95 \%$ credibility intervals (CrIs). We evaluated the relative ranking probability of each strategy and obtained the hierarchy of competing interventions using surface under the cumulative ranking curve (SUCRA). ${ }^{[20]}$ We employed the nodesplitting method to assess the presence of inconsistency. ${ }^{[21]}$

\subsection{Quality of evidence}

Two researchers (K.J and S.Q) independently evaluated the quality of each pair of comparison. The Grading of Recommendations, Assessment, Development and Evaluation (GRADE) methodology ${ }^{[22]}$ was performed to rate the quality of evidence. In this approach, direct evidence from RCTs starts at high quality and can be downgraded based on risk of bias, indirectness, imprecision, inconsistency (or heterogeneity) and publication bias to levels of moderate, low and relatively low quality. ${ }^{[23]}$

\section{Result}

\subsection{Literature search and study characteristics}

The primary literature search yielded 1241 citations in total, of which 675 articles met the criteria that identified according to their titles and abstracts. The study selection process is shown in Figure 1. Among these consequences, 130 studies were screened for the inclusion after the full test analyses. 113 articles were

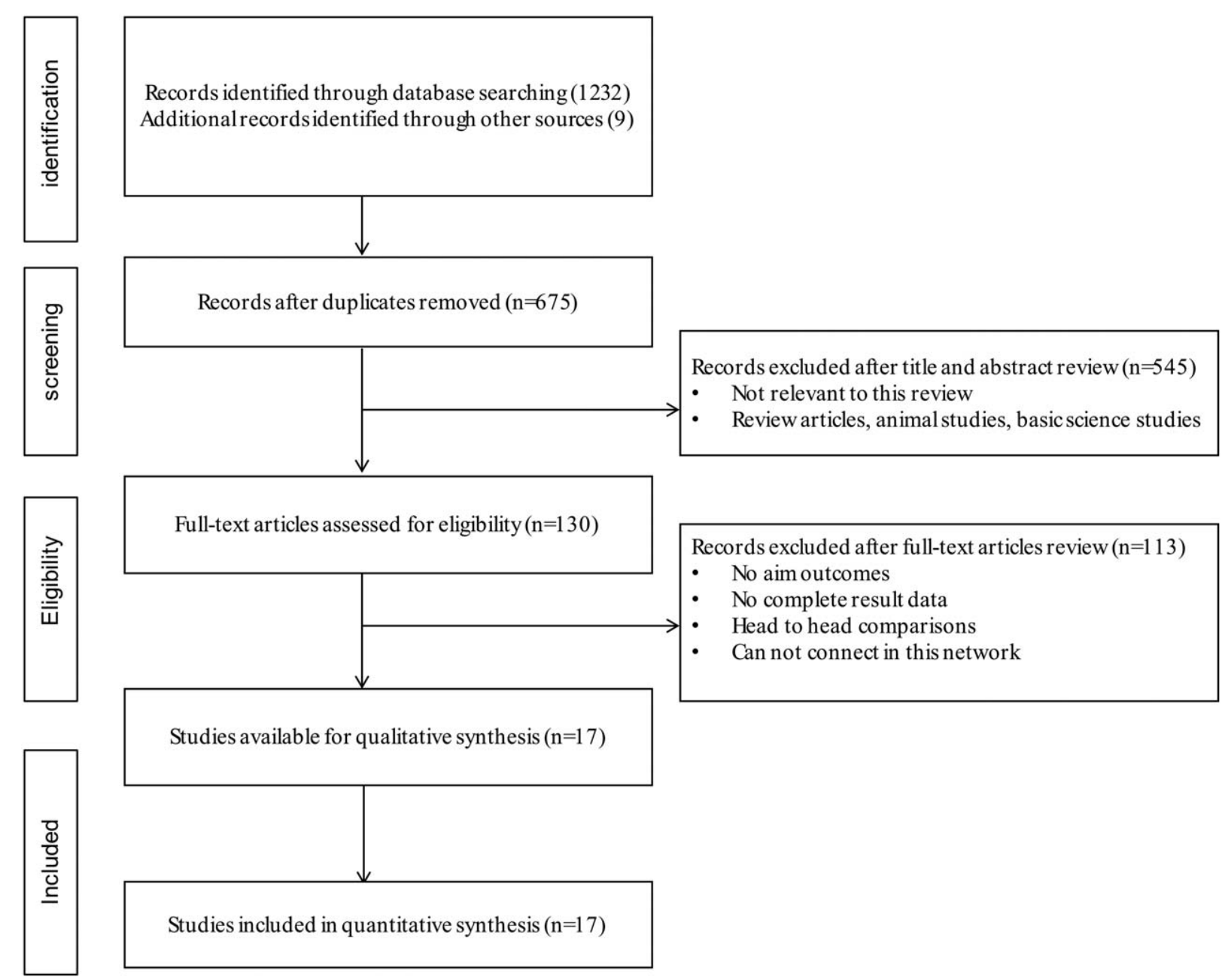

Figure 1. Flowchart of studies inclusion. 
excluded for the reason of researches with single-armed experimental group, no randomized study design, crossover or quasi-randomized studies, no relevant outcome reported and no relevant treatment. In the end, 17 eligible articles were left to fit our analysis. ${ }^{[924-39]}$ Table 1 summarizes the characteristics of these 17 RCTs covering 5739 adults aged from 18-77. All participants in the trials were diagnosed premature ejaculation or with IVELT of about $0.5-1.5 \mathrm{~min}$ more than 1 year. Overall, 7 single medication (paroxetine, dapoxetine, fluoxetine, sertraline, tadalafil, sildenafil, placebo) and 5 combined medications were included (tadalafil plus sildenafil, dapoxetine plus mirodenafil, tadalafil plus fluoxetine, paroxetine plus sildenafil, sildenafil plus fluoxetine). The network plot had a polygonal network configuration with mixed connections (Fig. 2 and online supplementary appendix 2, http://links.lww.com/MD/C673).

\subsection{Quality assessment}

The details of quality assessment are as measured by the Cochrane Collaboration risk-of-bias tool (see online supplementary appendix 3, http://links.lww.com/MD/C673). Quality assessment were measured by Cochrane risk-of-bias tool. Details of these 17 studies are shown in Figure 3. Five studies ${ }^{[24,26,28,33,35]}$ showed low risk in random sequence generation, and 4 studies ${ }^{[9,26,29,35]}$ showed low risks in allocation concealment. Risk of participant blinding is high in 3 studies $^{[24,25,32]}$ and low in 9 studies..$^{[9,26-30,33-35]}$ Only 1 study ${ }^{[9]}$ demonstrated high risk of outcome assessment blinding, while 9 studies ${ }^{[24,26-30,33-35]}$ showed low risk. Four studies $^{[24,25,29,34]}$ were found low risk in incomplete outcome data, accompanied with 9 studies ${ }^{[9,26-28,30-33,35]}$ in high risk. High risk in selective reporting were discovered in 7 studies. ${ }^{[17-19,25-26,28-29]}$ Risk of other source of bias were all low in 13 studies. ${ }^{[9][24-35]}$

\section{Table 1}

Study characteristic and baseline characteristics of included studies.

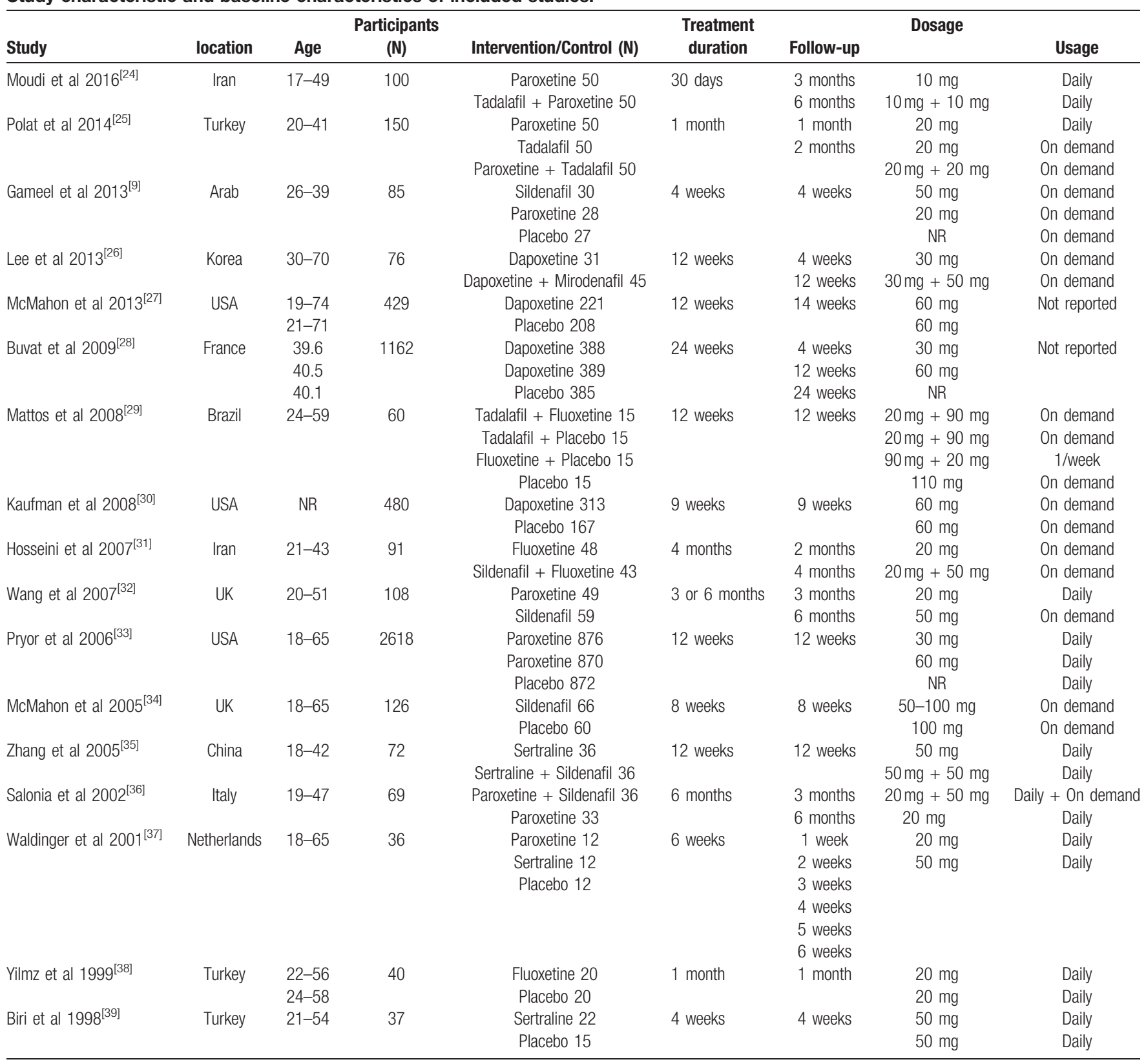



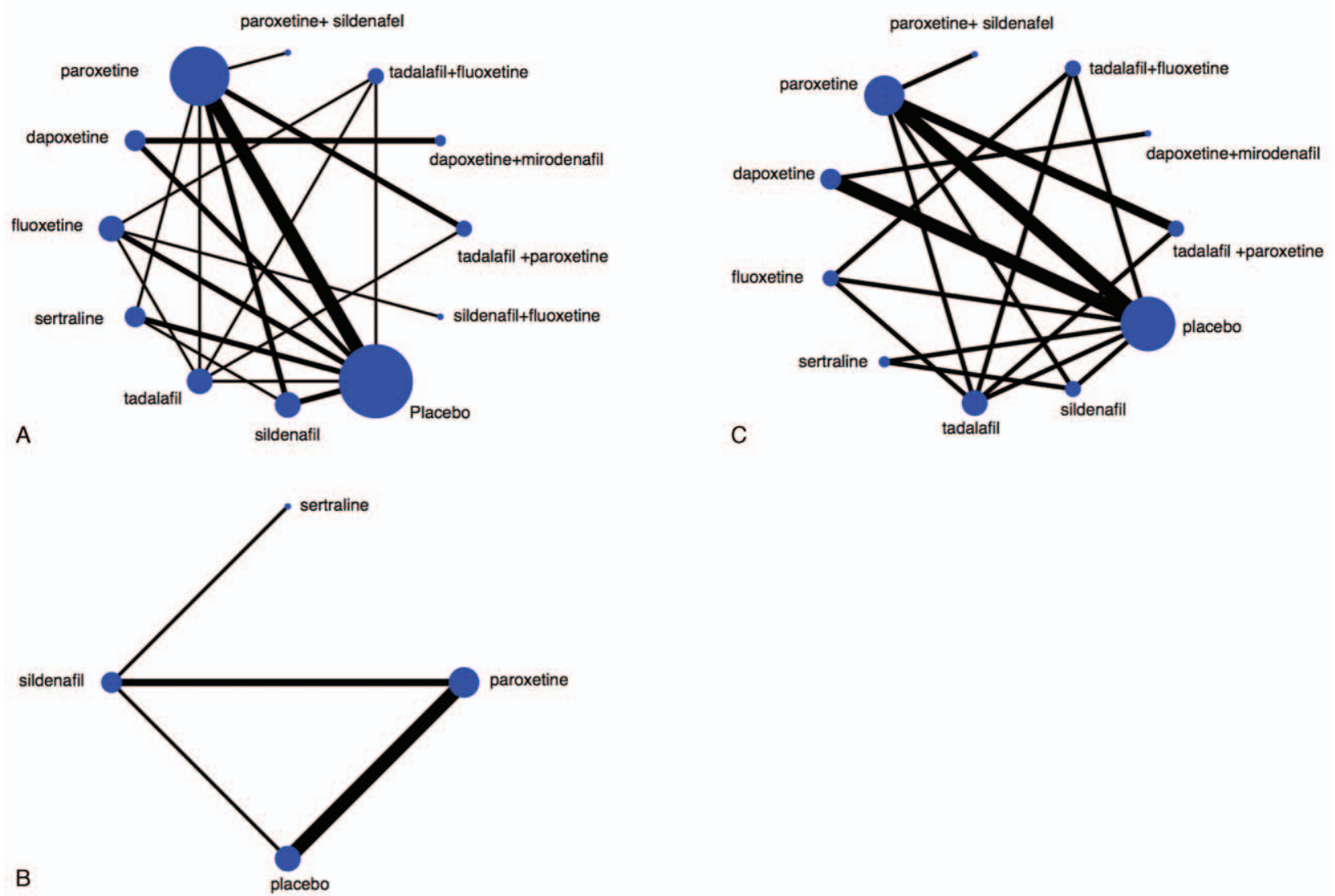

Figure 2. Evidence network for (A) IVELT; (B) Satisfactory score; (C) Adverse effects. The size of each circle (node) is proportional to the number of randomly assigned patients and indicates sample size. The number of randomised-controlled trials (RCTs) that contributed to each direct comparison is indicated on the line between nodes.

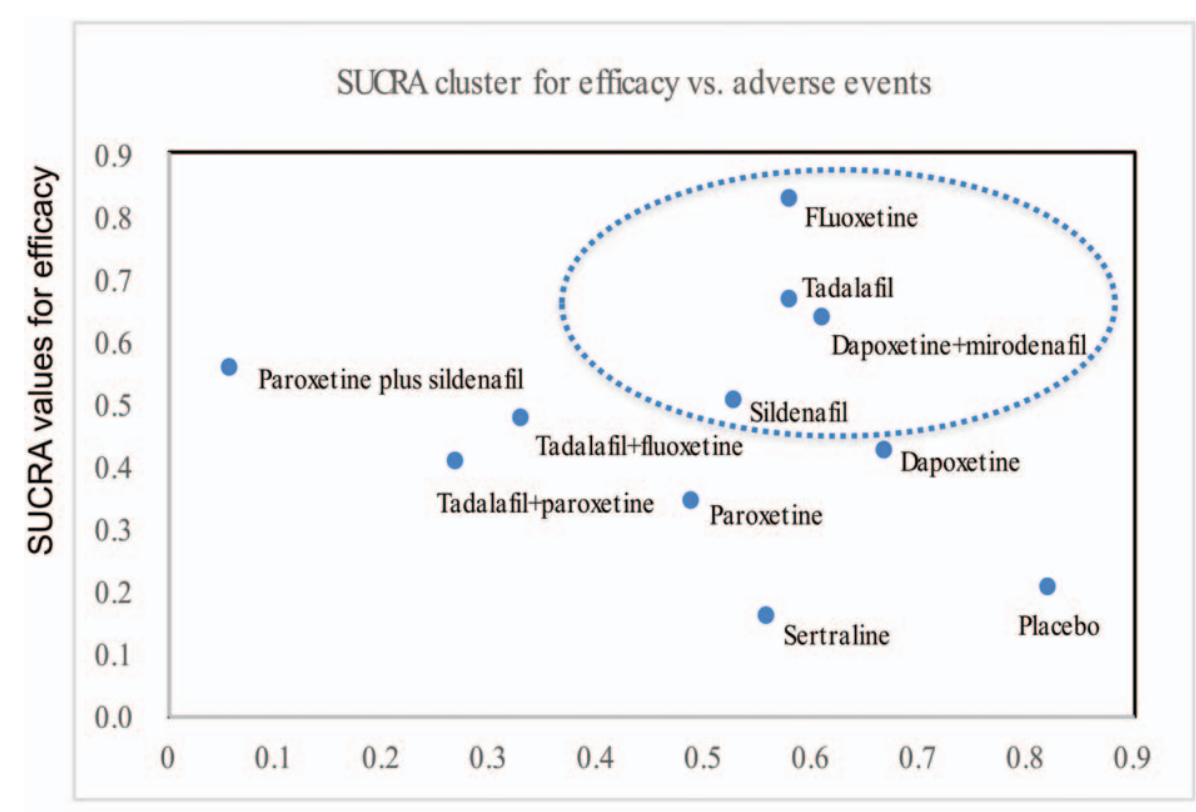

SUCRA values for adverse events

Figure 3. Scatterplot including SUCRA value for efficacy and SUCRA value for AEs. 
In addition, 4 studies ${ }^{[36-39]}$ were difficult to assess the bias for the reason of lacking complete methodology descriptions.

3.2.1. IVELT. Seventeen studies ${ }^{[9,24-39]}$ including 5739 patients were included in this analysis. In the pairwise meta-analysis, compared with placebo, paroxetine demonstrated a significant increase of IVELT (MD 0.89, 95\% CI 0.86 to $0.93, P<.001$ ). But there was a high heterogeneity with the $\mathrm{I}^{2}$ index of $99 \%$. Another significant improvement of IVELT was found in sertraline compared with placebo (MD 0.56, 95\% CI: 0.50 to 0.62 , $P<.001)$. Considering the result of NMA, paroxetine and sertraline both showed no significant difference compared with placebo (MD 0.68, 95\% CrI -0.33 to 1.89 ; MD -0.19, $95 \%$, CrI -1.49 to 1.68$)$. Two comparisons without contrast of pairwise led to significant difference in therapy: paroxetine plus sildenafil versus placebo (MD 1.75, 95\% CrI 0.05 to 3.78) and sildenafil versus sertraline (MD 1.63, 95\% CrI 0.10 to 2.79). Moreover, sildenafil was significantly superior to the placebo (MD 1.43, $95 \%$ CrI 0.003 to 2.81 ); However, in the pairwise meta-analysis, no significant difference was witnessed (MD 1.38, 95\% CrI - 0.55 to 2.32). No significant differences were found between other agents. SUCRA analysis suggested sildenafil plus fluoxetine $(85 \%)$, fluoxetine $(80 \%)$, tadalafil $(64 \%)$, dapoxetine plus mirodenafil $(60 \%)$, paroxetine plus sildenafil $(58 \%)$ and sildenafil $(53 \%)$ as the 6 treatments with the highest probability of IVELT amelioration (see online supplementary appendix 4, http://links.lww.com/MD/C673).

\subsection{Satisfactory score}

Five studies ${ }^{[9,30,32-33,36]}$ including 3363 patients were incorporated in the analysis. In the pairwise meta-analysis, the therapy benefit of sildenafil was significantly better than paroxetine (MD $0.82,95 \%$ CI 0.54 to $1.11, P=.038$ ). Compared with placebo, paroxetine could satisfy patients more obviously (MD $0.56,95 \%$ CI 0.48 to $0.63, P=.09)$. Correspondingly, in the NMA, sertraline, sildenafil and paroxetine were associated with significantly better satisfactory score than placebo (MD 4.62, $95 \%$ CrI 3.74 to 5.72 ; MD 1.52 , 95\% CrI 1.10 to 2.41 ; MD $0.61,95 \%$ CrI 0.38 to 1.07$)$. Compared with paroxetine, sertraline and sildenafil were associated significant improvement (MD 4.01, 95\% CrI 3.16 to 5.08; MD 0.91, 95\% CrI 0.50 to 1.56). Sildenafil demonstrated significant benefit to sertraline (MD 3.10, 95\% CrI 2.37 to 3.83). The SUCRA were $100 \%$, $66 \%, 33 \%, 0 \%$ for sertraline, sildenafil, paroxetine and placebo, respectively.

\subsection{Adverse events}

Considering pairwise meta-analysis, paroxetine is inferior to placebo (OR 6.10, 95\% CI 2.81 to $13.25, P<.001$ ). Dapoxetine had a higher possibility of causing side effects compared with placebo (OR 2.40, 95\% CI 2.00 to $2.89, P=.007$ ). Seventeen studies consisting of 5379 patients were included in the NMA. Compared with tadalafil plus paroxetine, dapoxetine showed significantly less AEs (OR 0.23, 95\% CI 0.02 to 0.96 ); Placebo demonstrated obviously lower risk of AEs comparing to paroxetine, sildenafil and paroxetine plus sildenafil (OR 0.20, $95 \%$ CI 0.05 to 0.52 ; OR $0.23,95 \%$ CI 0.04 to 0.80 ; OR 0.45 , $95 \%$ CI 0.01 to 0.92$)$. Paroxetine plus sildenafil (6\%), dapoxetine plus mirodenafil $(27 \%)$ and tadalafil plus paroxetine $(33 \%)$ were the 3 interventions with the highest probability of causing AEs.

\subsection{SUCRA cluster}

Figure 3 shows a scatterplot including SUCRA value for efficacy on the $y$-axis and SUCRA value for AEs on the $x$-axis. Cluster analysis demonstrates the division of treatments into 2 distinct groupings. One cluster of interventions, which includes fluoxetine, tadalafil, sildenafil and dapoxetine plus mirodenafil, has higher SUCRA values for both outcomes compared with the other grouping. (Fig. 3)

\subsection{Network consistency}

There was no inconsistency in the NMA estimates when we used the node-splitting approach and the differences between direct and indirect estimates in closed loops were insignificant which unable the assessment of network coherence. The total residual deviance for IVELT improvement $(37.2, \mathrm{df}=36.4)$, satisfactory score $(12.7, \mathrm{df}=12.3)$ and adverse events $(28.5, \mathrm{df}=26.7)$ implied a good model fit.

\section{Discussion}

Several causes, including organic and psychogenic factors, are speculated to the possible mechanisms of PE, but the exact causes are still unclear. According to animal and human psychopharmacological studies, central serotonergic neurotransmission is related to lifelong $\mathrm{PE},{ }^{[9]}$ for example, 5-hydroxytryptamine-2C receptor hyposensitivity and/or $1 \mathrm{~A}$ receptor hypersensitivity, as proposed mechanisms. ${ }^{[38-42]}$ There is no standard therapy because of no exact mechanism. The results of this NMA demonstrate important differences in IVELT and AEs between various interventions for PE. Our findings suggest a possible IVELT advantage of sildenafil and paroxetine plus sildenafil compared to placebo. Compared 1 treatment to the other, no differences were found. Notably, their benefits must be weighed against possible harms or adverse effects.

McMahon et al reported a possible explain of IVELT increase: The reduction in post-ejaculatory refractory time and confidence observed among men may lead to improved IVELT in the longer term. ${ }^{[34]}$ For the same problem, McMahon CG suggested that the proposed mechanisms of action of sildenafil in managing PE was relevant to improving the erection and down-regulating the erectile threshold, which making the arousal easier. ${ }^{[41]}$ On the contrary, Abdel-Hamid et al contributes this phenomenon to the pathway of nitric oxide (NO)-cGMP, through suppressing the contraction of the seminal vesicle, vas deferens, prostate and urethra and decreasing the control of the central sympathetic to smooth muscle, thus increases the IVELT. ${ }^{[25]}$ In a well-designed, randomized, double-blind, placebo-controlled study, compared with placebo, sildenafil has following advantages: enhancing the consciousness of ejaculatory control, sexual satisfaction and confidence; reducing depression, anxiety and the duration of reaching a second erection after ejaculation. ${ }^{[34]}$ Abdel-Hamid IA et al estimated that sildenafil can increase the baseline IVELT as much as 5 times sertraline, ${ }^{[43]}$ our NMA showed consistent result that sildenafil was associated with 1.63 times IVELT than sertraline. There are several possibilities that may cause the difference in multiple: patient selection, baseline IVELT, and drug dosages. One study showed that sildenafil is superior than the all other treatment methods in the aspects of IVELT control and overall satisfaction $(P<.0001) .{ }^{[43]}$ In the randomized experiment conducted by Gammel, on-demand use of sildenafil provided the best overall sexual satisfaction scores, and exerted a better outcome than paroxetine and local penile anaethetics. ${ }^{[9]}$ But our 
NMA showed differently from the result: Sertraline ranked superior to Sildenafil. However, satisfaction criteria varied in various studies, besides, there is no exact and accurate criterion to measure satisfaction of the partner. Among the studies, various methods were used to assess satisfaction. For the reason that satisfaction is a subjective feeling, a standard assessment should be made to defined in order to determine patient post-treatment satisfactory and partner satisfactory.

Sildenafil can cause some tolerant side effects, including nausea, diarrhea, dizziness, headache, insomnia, dyspepsia, and so on, of which nausea and dizziness are the main AEs leading to withdrawal in 1 meta-analysis. ${ }^{[10]}$ All the RCTs included in the NMA showed that SSRI, PDE5I and other medication caused more side effects than placebo. In accordance with this conclusion, our NMA work demonstrated that sildenafil caused more side effects than placebo.

Waldinger et al reported that SSRI was demonstrated to be effective in relieving PE, with paroxetine bringing about the most remarkable delay in ejaculation compared with baseline values, probably due to activation of the 5-HT2c receptor that then inhibit the function of the 5-HT1a receptors or keeps a dynamic balance between the 2-receptor functions (5-HT1a and 5-HT2c). ${ }^{[40-41]}$ In our NMA, the combination of paroxetine plus sildenafil was associated with 1.75 times IVELT than placebo. However, the combination therapy was comparable to paroxetine or sildenafil alone in promoting the IVELT. In the study conducted by Salonia et $\mathrm{al}^{[37]}$ the result estimated that the IVELT improved in patients using combined therapy, which is corresponding to our NMA results. Three previous meta-analysis ${ }^{[12-14]}$ all showed that therapy with SSRIs plus PDE5i for PE was associated with a significantly greater increase in mean IVELT compared with SSRI or PDE5i alone. The probable reason is that the mechanism of SSRI differs from that of PDE5i, 2 types of medicine take effect in different targets, and pharmacodynamics of them can't influence each other. Owing to this, the side effects are more likely to occur, which is corresponded with our NMA. Another meta-analysis recommended dapoxetine at $30 \mathrm{mg}$ as the first-line agent. ${ }^{[4]}$ However, paroxetine plus sildenafil showed more IVELT than dapoxetine in the NMA, but the author negate the result for lack of pair-wise comparison of the 2 therapies. In the overall consideration, sildenafil plus paroxetine precedes monotherapy except for a little more tolerated side effects. IVELT and side effects have to be considered when using combination of 2 drugs.

There are some limitations in our NMA: First, the criterion of PE is not defined, causing the baseline of IVELT different. This gap may lead to the improvement of IVELT more notable, exaggerating the efficacy of medicine or making the result inaccuracy. The second limitation is that there are not enough studies, and non-English articles were excluded, causing high risks of typeI and typeII errors. Third, the duration of treatment and follow-up are various, no clear data has determined the best duration. And short-term of treatment may conceal the efficacy of some medicine. Fourth, the dosage and usage can affect the therapeutic result and occurrence rate of side effects. In 1 metaanalysis, ${ }^{[9]}$ doubling dosage or on demand use improve IVELT more obviously, causing more side effects at the same time. Furthermore, it is unclear whether the combination of paroxetine plus sildenafil can still be tolerated in the long-term treatment, and whether it can cause new complications. More researches are needed to make up for the deficiency above.

\section{Conclusion}

IVELT and side effects have to be considered when using combination of 2 drugs. Our study indicated that although paroxetine plus sildenafil and sildenafil alone both demonstrated significant IVELT benefit compared with placebo, significant increase of AEs risk was also observed. Furthermore, sildenafil alone was superior to sertraline in efficacy with comparable tolerability, but no conclusion can be drawn in terms of the comparison of the combined treatment based on current evidence. Further investigations focusing on long-term effectiveness and acceptability of treatments, and investigate the optimal timing and thresholds for treatments are warranted to draw a final conclusion.

Table 2

\section{Table 2}

Statistically significant results of network meta-analysis.

\begin{tabular}{|c|c|c|c|c|c|c|c|}
\hline Comparisons & $\begin{array}{l}\text { No. of } \\
\text { studies }\end{array}$ & $\begin{array}{c}\text { No. of } \\
\text { participants }\end{array}$ & $\begin{array}{l}\text { Pairwise meta-analysis } \\
\text { mean difference/odds } \\
\text { ratios }(95 \% \mathrm{Cl})\end{array}$ & $\begin{array}{c}\text { Network meta-analysis } \\
\text { mean difference/odds ratios } \\
\text { (95\% Crl) }\end{array}$ & $\begin{array}{l}\text { Heterogeneity } \\
\qquad\left.\right|^{2}\end{array}$ & $P$ value & $\begin{array}{l}\text { Quality of } \\
\text { evidence }\end{array}$ \\
\hline \multicolumn{8}{|l|}{ IVELT } \\
\hline Sildenafil vs Sertraline ${ }^{[36]}$ & 1 & 36 vs 36 & - & $1.63(0.01$ to 2.79$)$ & - & - & Low \\
\hline Sildenafil vs Placebo & 2 & 96 vs 87 & $1.38(-0.55$ to 3.32$)$ & 1.43 (0.003 to 2.81$)$ & $0 \%$ & .85 & Low \\
\hline \multicolumn{8}{|l|}{ Satisfactory Score } \\
\hline Paroxetine vs Placebo ${ }^{[9,30,33]}$ & 4 & 1217 vs 1066 & $0.56(0.48$ to 0.63$)$ & 0.61 (0.38 to 1.07$)$ & $54 \%$ & .09 & Moderate \\
\hline Sildenafil vs Sertraline ${ }^{[36]}$ & 1 & 36 vs 36 & - & $3.10(2.37$ to 3.83$)$ & - & - & Low \\
\hline Sertraline vs Placebo & 0 & - & - & 4.62 (3.74 to 5.72 ) & - & - & Low \\
\hline Sildenafil vs Placebo ${ }^{[9]}$ & 1 & 30 vs 27 & - & $1.52(1.10$ to 2.41$)$ & - & - & Low \\
\hline \multicolumn{8}{|l|}{ Adverse effect } \\
\hline Dapoxetine vs Tadalafil plus Paroxetine & 0 & - & - & 0.23 (0.02 to 0.96$)$ & - & - & Low \\
\hline
\end{tabular}

95\% Cl=95\% Confidence Intervals; 95\% Crl=95\% Credible Intervals; , IVELT = intravaginal ejaculation latency time; Quality of evidence as judged based on the Grading of Recommendations Assessment, Development and Evaluation (GRADE) approach. First, we rated quality of evidence for direct comparisons; second, we rated quality of evidence for indirect estimates (starting at the lowest rating of the 2 pairwise direct estimates that contribute as first-order loops to the indirect estimate, which can be rated down further for imprecision or intransitivity), and then third, rating the quality of evidence for the network combining direct and indirect estimates. In this step, if direct and indirect estimates from second-order comparisons are similar, the higher of the ratings was assigned to the network meta-analysis estimates. 


\section{Acknowledgments}

The authors would like to thank staff at the Chinese Evidencebased Medicine Centre, State Key Laboratory of Biotherapy, West China Hospital, Sichuan University for logistical support of this systematic review.

\section{Author contributions}

QW and LY conceived this review. KJ, XT, SQ, and LHD identified reports of trials and extracted data. KJ provided statistical advice and SQ did all statistical analyses. JKL and LHD checked for statistical inconsistency and interpreted data. LY, $\mathrm{XT}, \mathrm{YGB}$, and SQ contributed to data interpretation. KJ drafted the report and all other authors (QW, LY, SQ, LHD, ZHT, YGB, and JKL) critically reviewed the article. All authors read and approved the final manuscript.

Conceptualization: Yige Bao and Lu Yang.

Data curation: Xiang Tu.

Formal analysis: Jiakun Li.

Funding acquisition: Qiang Wei.

Methodology: Shi Qiu.

Project administration: Lu Yang.

Resources: Qiang Wei.

Software: Yige Bao, Qiang Wei.

Validation: Lu Yang.

Visualization: Yige Bao.

Writing - original draft: Linghui Deng.

Writing - review \& editing: Kun Jin and Qiang Wei.

\section{References}

[1] Porst H, Montorsi F, Rosen RC, et al. The premature ejaculation prevalence and attitudes (pepa) survey: prevalence, comorbidities, and professional help-seeking. Eur Urol 2007;51:816-24.

[2] Serefoglu EC, McMahon CG, Waldinger MD, et al. An evidence-based unified definition of lifelong and acquired premature ejaculation: report of the second international society for sexual medicine ad hoc committee for the definition of premature ejaculation. Sex Med 2014;2:41-59. DOI:10.1002/sm2.27.PMID:25356301.

[3] Washington, DC. American Psychiatric Association. Diagnostic and Statistical Manual of Mental Disorders, 4th ed. Text Revision. (DSMIVTR). American Psychiatric Association, 2000:554

[4] Symonds T, Roblin D, Hart K, et al. How does premature ejaculation impact a man's life? J Sex Marital Ther 2003;29:361-70.

[5] Rowland D, Perelman M, Althof S, et al. Self-reported premature ejaculation and aspects of sexual functioning and satisfaction. J Sex Med 2004;1:225-32.

[6] Waldinger MD. Lifelong premature ejaculation: from authority-based to evidence-based medicine. BJU Int 2004;93:201-7.

[7] Waldinger MD, Zwinderman a H, Schweitzer DH, et al. Relevance of methodological design for the interpretation of efficacy of drug treatment of premature ejaculation: a systematic review and meta-analysis. Int J Impot Res 2004;16:369-81.

[8] Balon R. Antidepressants in the treatment of premature ejaculation. J Sex Marital Ther 1996;22:85-96.

[9] Gameel TA, Tawfik AM, Abou-Farha MO, et al. On-demand use of tramadol, sildenafil, paroxetine and local anaesthetics for the management of premature ejaculation: a randomised placebo-controlled clinical trial. Arab J Urol 2013;11:392-7.

[10] Li J, Yuan H, Bai Y, et al. Dapoxetine for premature ejaculation: an updated meta-analysis of randomized controlled trials. Clin Ther 2014;36:2003-14.

[11] Yue FG, Dong L, Hu TT, et al. Efficacy of dapoxetine for the treatment of premature ejaculation: a meta-analysis of randomized clinical trials on intravaginal ejaculatory latency time, patient-reported outcomes, and adverse events. Urology 2015;85:856-61.

[12] Bai Y, Pu C, Han P, et al. Selective serotonin reuptake inhibitors plus phosphodiesterase 5 inhibitors for premature ejaculation: a systematic review and meta-analysis. Urology 2015;86:758-65.
[13] Men C, Yu L, Yuan H, et al. Efficacy and safety of phosphodiesterase type 5 inhibitors on primary premature ejaculation in men receiving selective serotonin reuptake inhibitors therapy: a systematic review and meta-analysis. Andrologia 2016;48:978-85.

[14] Sun Y, Luo D, Yang L, et al. Efficacy of phosphodiesterase-5 inhibitor in men with premature ejaculation: a new systematic review and meta-analysis. Urology 2015;86:947-54. doi:10.1016/j.urology.2015. 06.051.

[15] Yang L, Qian S, Liu H, et al. Role of tramadol in premature ejaculation: a systematic review and meta-analysis. Urol Int 2013;91:197-205. doi:10.1159/000348826.

[16] Castiglione F, Albersen M, Hedlund P, et al. Current pharmacological management of premature ejaculation: a systematic review and metaanalysis. Eur Urol 2015;1-3.

[17] Hutton B, Salanti G, Caldwell DM, et al. The PRISMA extension statement for reporting of systematic reviews incorporating network meta-analyses of health care interventions: checklist and explanations. Ann Intern Med 2015;162:777-84.

[18] Higgins JPT, Green S. Cochrane Handbook for Systematic Reviews of Interventions Version 5.1.0 [updated March 2011]. In: The Cochrane Collaboration. 2011. Table 7.7.a: Formulae for combining groups.

[19] Salanti G, Higgins JP, Ades A, et al. Evaluation of networks of randomized trials. Stat Methods Med Res 2007;17:279-301.

[20] Higgins JPT, Jackson D, Barrett JK, et al. Consistency and inconsistency in network meta-analysis: concepts and models for multi-arm studies. Res Synth Methods 2012;3:98-110.

[21] Higgins JPT, Thompson SG, Deeks JJ, et al. Measuring inconsistency in meta-analyses. BMJ Br Med J 2003;327:557-60.

[22] Guyatt GH, Oxman AD, Vist GE, et al. GRADE: An emerging consensus on rating quality of evidence and strength of recommendations. Chin J Evid-Based Med 2009;9:8-11.

[23] Salanti G, Giovane C. Del, Chaimani A, et al. Evaluating the quality of evidence from a network meta-analysis. PLoS One 2014;9.

[24] Moudi E, Kasaeeyan AA. Comparison between tadalafil plus paroxetine and paroxetine alone in the treatment of premature ejaculation. Nephrourol Mon 2016;8:e32286.

[25] Polat EC, Ozbek E, Otunctemur A, et al. Combination therapy with selective serotonin reuptake inhibitors and phosphodiesterase-5 inhibitors in the treatment of premature ejaculation. Andrologia 2015;47:487-92.

[26] Lee WK, Lee SH, Cho ST, et al. Comparison between on-demand dosing of dapoxetine alone and dapoxetine plus mirodenafil in patients with lifelong premature ejaculation: prospective, randomized, double-blind, placebo-controlled, multicenter study. J Sex Med 2013;10:2832-41.

[27] Mcmahon CG, Giuliano F, Dean J, et al. Efficacy and safety of dapoxetine in men with premature ejaculation and concomitant erectile dysfunction treated with a phosphodiesterase type 5 inhibitor: randomized, placebo-controlled, phase III study. J Sex Med 2013;10:2312-25.

[28] Buvat J, Tesfaye F, Rothman M, et al. Dapoxetine for the treatment of premature ejaculation: results from a randomized, double-blind, placebo-controlled phase 3 trial in 22 countries. Eur Urol 2009;55: 957-68.

[29] Mattos RM, Marmo Lucon A, Srougi M. Tadalafil and fluoxetine in premature ejaculation: prospective, randomized, double-blind, placebocontrolled study. Urol Int 2008;80:162-5.

[30] Kaufman JM, Rosen RC, Mudumbi RV, et al. Treatment benefit of dapoxetine for premature ejaculation: results from a placebo-controlled phase III trial. BJU Int 2009;103:651-8.

[31] Hosseini MM, Yarmohammadi H. Effect of fluoxetine alone and in combination with sildenafil in patients with premature ejaculation. Urol Int 2007;79:28-32.

[32] Wang WF, Wang Y, Minhas S, et al. Can sildenafil treat primary premature ejaculation? A prospective clinical study. Int J Urol 2007;14:331-5.

[33] Pryor JL, Althof SE, Steidle C, et al. Efficacy and tolerability of dapoxetine in treatment of premature ejaculation: an integrated analysis of two double-blind, randomised controlled trials. Lancet 2006;368:929-37.

[34] McMahon CG, Stuckey BGA, Andersen M, et al. Efficacy of sildenafil citrate (viagra) in men with premature ejaculation. J Sex Med 2005;2:368-75.

[35] Waldinger MD, Zwinderman AH, Olivier B. Antidepressants and ejaculation: a double-blind, randomized, placebo-controlled, fixed-dose study with paroxetine, sertraline, and nefazodone. J Clin Psychopharmacol 2001;21:293-7. 
[36] Zhang X, Wang Y, Huang X, et al. Comparison between sildenafil plus sertraline and sertraline alone in the treatment of premature ejaculation. Zhonghua Nan Ke Xue 2005;11:520-2, 525.

[37] Salonia A, Maga T, Colombo R, et al. A prospective study comparing paroxetine alone versus paroxetine plus sildenafil in patients with premature ejaculation. J Urol 2002;168:2486-9.

[38] Yilmaz U, Tatlisen A, Turan H, et al. The effects of fluoxetine on several neurophysiological variables in patients with premature ejaculation. J Urol 1999;161:107-11.

[39] Biri H, Isen K, Sinik Z, et al. Sertraline in the treatment of premature ejaculation: a double-blind placebo controlled study. Int Urol Nephrol 1998;30:611-5.

[40] Waldinger MD, Hengeveld MW, Zwinderman AH, et al. Effect of SSRI antidepressants on ejaculation: a double-blind, randomized, placebo- controlled study with fluoxetine, fluvoxamine, paroxetine, and sertraline. J Clin Psychopharmacol 1998;18:274-81.

[41] Waldinger MD, Berendsen HHG, Blok BFM, et al. Premature ejaculation and serotonergic antidepressants-induced delayed ejaculation: the involvement of the serotonergic system. Behav Brain Res 1998;92: $111-8$.

[42] Waldinger MD. The neurobiological approach to premature ejaculation. J Urol 2002;168:2359-67.

[43] Abdel-Hamid IA, El Naggar EA, El Gilany AH. Assessment of as needed use of pharmacotherapy and the pause-squeeze technique in premature ejaculation. Int J Impot Res 2001;13:41-5.

[44] Pharmacological interventions for premature ejaculation: a mixed treatment comparison network meta-analysis of randomized clinical Trials. Int J Impot Res 2018;30:215-23. 